\title{
Overcoming Errors in Fraction Computation by Emphasizing Unit Fractions, Length Drawings, and Student Explanations
}

\author{
Karen C. Fuson \\ School of Education and Social Policy, Northwestern University, United States \\ *Corresponding Author: fuson@northwestern.edu
}

Copyright $\bigcirc 2019$ by authors, all rights reserved. Authors agree that this article remains permanently open access under the terms of the Creative Commons Attribution License 4.0 International License

\begin{abstract}
This paper addresses the perceived opacity of fraction computation by summarizing results of classroom design experiments focused on making fraction concepts and computation meaningful to students and teachers. A nurturing Math Talk classroom environment, in which students made and explained math drawings supported sense-making by students and teachers. Students were able to make drawings of length models connected to symbolic computations for all fraction operations, and most students were able to explain their thinking. Correct performance on computations was considerably higher than that of U.S. students using traditional textbooks and was more comparable to performance of East Asian students. The paper shows the length drawings for each fraction operation and summarizes the nature of students' errors and how to overcome these. Length drawings (bar and number line models) connected to fraction symbols and words can focus students on unit fractions in all operations. Important difficulties to overcome are that fraction notation does not differentiate the roles of the numerator and denominator, and the notation shows the number but not the size of unit fractions. Multiplying fractions by multiplying each unit fraction and not the whole fraction magnitude is a general method, and division of fractions can begin by dividing numerators and denominators.
\end{abstract}

Keywords Fractions, Fraction Computation, Rational Numbers, Fraction Bars, Fraction Number Line, Division as Finding the Unknown Factor

\section{Introduction}

Over thirty years ago, students in the United States performed poorly on items assessing fraction computation $[1,2]$, and students in East Asian countries performed better but still had areas of considerable weakness [1]. A recent paper [3] indicates that this is still the case in the United States: "Poor understanding of rational number arithmetic is common not only among students but also among teachers (p206)" and goes on to say "One difficulty is that rational number arithmetic procedures are often opaque (p207)." This paper addresses the perceived opacity of fraction computation by summarizing results of a series of classroom design experiments focused on making fraction concepts and fraction computation meaningful to students and teachers. These experiments began in single classrooms where one of the designers worked with a teacher and then moved to teaching experiments in which teachers taught using curricular materials written by the design team. Materials and methods and then overall results are summarized, and then specific opacities and learning difficulties are addressed along with what the teaching experiments found to be effective approaches in overcoming these difficulties. These are summarized as Design Principles in the discussion.

Small whole numbers are composed from single units of things. We compare, add/subtract, and multiply/divide single units and later larger composed units of tens, hundreds, etc. What are the units that compose to make fractions? Unit fractions are made from some whole that is divided ("fractured") into $n$ equal parts. One of these parts is the unit fraction $1 / n$, and it is these unit fractions that are compared, added/subtracted, and multiplied/divided in fraction computation. Many schools in the United States have traditionally used circles to show fractions and called these pizzas or pies to connect fractions to student's lives. But circles are difficult to divide into some parts (e.g., fifths, sevenths, tenths) and to further divide into subparts, comparison of parts is visually complex, and students make several kinds of errors when equal-dividing circles such as making fifths by dividing one fourth in half or drawing lines across the circles instead of making sectors from the center. Length models like fraction strips, drawn fraction 
bars, and fraction number lines do not have these disadvantages. Therefore, our design experiments used length models for all operations and sought ways to show how each operation uses unit fractions.

Our theoretical framework uses both a Piagetian constructivist model of learning and a Vygotskian socio-cultural model of teaching [see 4]. From our Piagetian perspective, we assume that students are continually interpreting their classroom experiences using their own conceptual structures as well as continually adapting their conceptual structures to their on-going classroom experiences. From our Vygotskian perspective, we assume that a major goal of school mathematics teaching is to assist learners in coming to understand and use cultural mathematics tools. One means of assistance is drawings, which are semiotic tools that can support sense-making both individually and in the classroom discourse about mathematical thinking. Therefore, our teaching approaches primarily used drawings that students could make and explain. A nurturing Math Talk classroom environment supported sense-making done by students and teachers [5].

\section{Materials and Methods}

\subsection{Participants}

Participants in the first study were 25 grade 5 (age 10 to 11) students in a classroom in a small mid-western multi-racial city with a considerable number of immigrants, a large minority of the students on free lunch, and a substantial number of students with highly-educated parents. This diversity was chosen to test the accessibility of our approach to a broad range of students.

Participants in the second study were 24 grade 5 students in a classroom in a different school in the same small city with the same range of students.

Participants in the third study were two classrooms in grade 3 (age 8 to 9), two classrooms in grade 4 (age 9 to 10), and one classroom in grade 5 (age 10 to 11 ) in a school in a large urban district. Almost all students were qualified for free lunch (an index of low family income), and most were from Spanish-speaking backgrounds. One classroom at each grade level had students not yet fluent in English, and one classroom in grades 3 and 4 had students fluent in English. Teaching materials were in English, and the bilingual teachers in the not-yet-English-fluent classrooms taught in English supplemented as needed with Spanish. Class sizes ranged from 31 to 37.

\subsection{Teachers and Teaching Materials}

In the first study, the second author of [6] co-taught the class with the regular classroom teacher to implement the intended approach and raise issues as needed. Lesson plans for the fifteen days' fraction unit were made initially and adapted as needed. For the second and third studies complete teacher and student materials were written and given to each teacher. The regular classroom teachers taught these units.

\subsection{Data Collection}

For the first study the second author of [6] videotaped each class, took notes, interviewed students, and collected overheads used for explanations by students and other student work. For the second and third studies, classes were visited occasionally by the author of this paper to watch math talk explanations of length drawings and to examine student work. The author talked by phone with teachers each weekend to answer any questions about the teaching for the previous or next week and to discuss how the student learning was going.

Data for student learning in all studies were students' daily performance in class including explanations of methods and written work, written homework, and a test given at the end of each unit. This test was comprised of numerical items comparable to and word problems identical to those given by Stigler, Lee, and Stevenson [1] to U.S. grade 5 students using traditional textbooks and to Japanese and Taiwanese grade 5 students. Other items were included to assess concepts not included in [1].

\section{Results}

\subsection{Student Performance}

For all three studies, examination of student written work during the unit indicated that most students were able to make length drawings for a given operation correctly, although some required help along the way with various steps. Complete explanations of representations in the length drawings related to fraction symbols were given by some to many students in every class either orally or in written form, and the other students could explain some of the steps clearly. All students did draw most of the steps on the length models correctly and gave accurate numerical labeling and symbolic computations.

Students in the first study did very well on the test of multiplication of fraction items given at the end of the unit [6]. More than $80 \%$ of our length-model students solved the numerical fraction computation problems correctly, compared to means of $20 \%, 21 \%$, and $14 \%$ in Japan, Taiwan, and the U.S. respectively. Our length-model students also did comparatively well on the word problems, with $63 \%$ correct answers compared to $54 \%, 49 \%$, and $20 \%$ respectively. On items for comparison $(<,>)$, addition, and subtraction with like unit fractions, our students were roughly comparable to the Japanese and Taiwanese students and considerably outperformed the U.S. students 
using traditional textbooks, with percent scores from $20 \%$ to $50 \%$ higher.

Grade 5 students in the second study scored at high levels similar to those in the first study.

Grade 5 students in the third study had scores about 10 percentage points lower than students in the first two studies, but they still considerably outperformed U.S. students using traditional textbooks [1]. Grade 3 and Grade 4 students in the third study learned to compose unit fractions, find equivalent fractions, and compare, add, and subtract like and unlike unit fractions (problems with like and unlike denominators). On these items students performed like Grade 5 students on composing unit fractions, finding equivalent fractions, and adding and subtracting like unit fractions. They had scores about 10 percentage points lower than the Grade 5 students on comparing, adding, and subtracting unlike unit fractions (unlike denominators).

\section{Build Fractions from Unit Fractions}

\section{Write the unit fractions for each whole. Next, shade the correct number of parts. Then show each shaded fraction as a sum of unit fractions.}

(9)

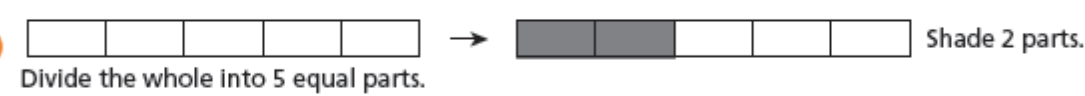

$$
\frac{1}{5}+\frac{1}{5}+\frac{1}{5}+\frac{1}{5}+\frac{1}{5} \quad \frac{1}{5}+\frac{1}{5}=\frac{2}{5}
$$

10

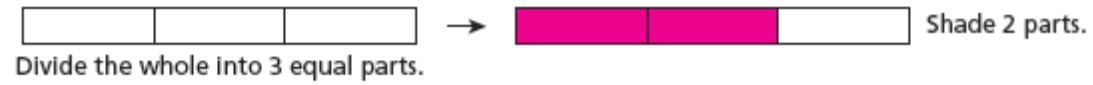

$$
\frac{1}{3}+\frac{1}{3}+\frac{1}{3} \quad \frac{1}{3}+\frac{1}{3}=\frac{2}{3}
$$

(11)

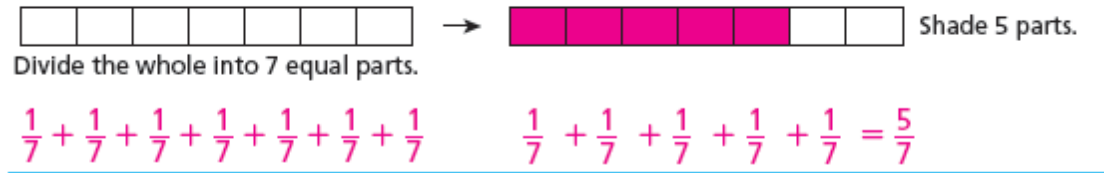

12

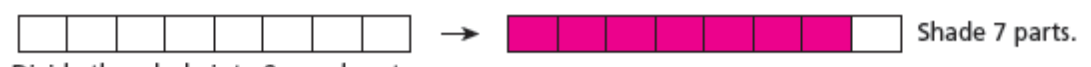

Divide the whole into 8 equal parts.

$$
\frac{1}{8}+\frac{1}{8}+\frac{1}{8}+\frac{1}{8}+\frac{1}{8}+\frac{1}{8}+\frac{1}{8}+\frac{1}{8} \quad \frac{1}{8}+\frac{1}{8}+\frac{1}{8}+\frac{1}{8}+\frac{1}{8}+\frac{1}{8}+\frac{1}{8}=\frac{7}{8}
$$

13

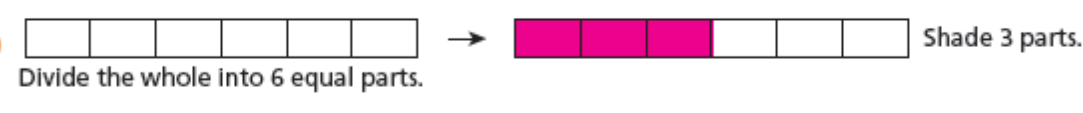

$$
\frac{1}{6}+\frac{1}{6}+\frac{1}{6}+\frac{1}{6}+\frac{1}{6}+\frac{1}{6}+\frac{1}{6}+\frac{1}{6}=\frac{3}{6}
$$

Figure 1. See unit fractions visually and in fraction notation $1 / n$ by showing division of the whole into $n$ equal parts and then in a separate drawing composing a fraction by shading or circling these unit fractions 


\subsection{Design Principles and Teaching Approaches to Overcome Typical Student Errors}

\subsubsection{See Unit Fractions Visually and in Fraction Notation} 1/n by Showing Division of the Whole into N Equal Parts and then in a Separate Drawing Composing Any Fraction by Shading or Circling These Unit Fractions

Fractions $a / b$ are frequently shown as $a$ of the $b$ parts shaded or otherwise indicated visually. Students then see easily the shaded and unshaded parts and write these as the fraction, seeing for example the fraction at the top right of Figure 1 as 2/3 ( 2 parts shaded and 3 parts unshaded). The $a$ in the fraction is embedded in the whole $b \times 1 / b$ unit fractions, making the total number of unit fractions difficult to see. We found that if students saw and made fraction length drawings in two steps on two drawings, they later became able to put together the shaded and unshaded parts mentally to make the unit fractions composing the one whole. So in all of the examples $a / b$ in Figure 1, the same one whole is divided into $b$ equal parts and then in the second drawing $a$ of them are shaded to make a particular fraction $a / b$ from the unit fractions.

It was also important for students to see and work with the written notation for a unit fraction as $1 / b$ so that the unit fraction conceptually and symbolically became a unit that could be added, subtracted, and compared using the meaning they saw or made on drawings as 1 of $b$ equal parts. Students wrote symbolic unit fractions $1 / b$ to label the parts of length models divided into $b$ equal parts (see the left column in Figure 1) and also wrote such symbolic unit fractions in a sum of the unit fractions composed to make a given fraction $a / b$ (see the right column in Figure 1). Students began to identify and use the pattern that one whole is composed of $b$ unit fractions $1 / b$ so that they could say how many more unit fractions made one whole, for example 5/7 needs 2 more unit fractions $1 / 7$ to make $7 / 7$ which is one whole. On all figures in this paper, red parts of drawings or symbols are parts that students drew or wrote.

Fractions are always part of some particular whole. To compare, add, or subtract unit fractions, they must be part of the same whole. This point was raised for students to discuss at the beginning of work on fractions when students were given strips of paper and folded them to make fractions. Half of the class had strips that were cut horizontally from paper and half had strips that were cut vertically from the same sized paper. Students folded their strips to make 2, 3, 4, 5, and 6 equal parts and labeled all of the parts with a unit fraction $1 / 2,1 / 3,1 / 4,1 / 5$, and $1 / 6$. One strip was labeled one whole. Students discussed patterns they saw, for example, the unit fractions became smaller as the number of unit fractions became larger. This inverse relationship is a key conceptual feature of fractions, and we will return to it several times. In this discussion students also raised questions such as, "Why is my $1 / 2$ bigger than his $1 / 2$ ?" In the classroom, there were two different lengths labeled $1 / 2$, and each was correctly labeled because they were both 1 of 2 equal parts. Students saw and explained that the fraction was $1 / 2$ of some whole, and that whole could vary. So the important issue of "which whole" became salient very early.

The English language uses almost the same words for unit fractions as for ordinal position: half (second for ordinal position), third, fourth, fifth, sixth, etc. These words provide no hint to the meaning of a fraction or of a unit fraction. These words also can be confusing for students who understand ordinal position: Fifth in line for tickets is not one of five equal parts. This is in contrast to fraction words in Chinese, which say for 3/5: of 5 parts, 3. This names the unit fraction first so students can visualize that one whole is divided into 5 parts before thinking about how many those unit fractions they will take to make a given fraction. To support this conceptual focus on the number of unit fractions and to give some sense of fractions resulting from division, we used in the classroom a number and the word fracture for the first equal-dividing step: Dividing into 3 equal parts was a 3 -fracture, and a 5 -fracture resulted in 5 equal parts, each $1 / 5$. Students readily used this language to describe their own length drawings and drawings such as in Figure 1 used in the classwork and homework. Fracture in ordinary English of course carries no meaning of equal parts; most real-world fractures result in unequal parts. But students seemed to easily add this constraint of equal parts, which was facilitated by drawings such as in Figure 1 and class explanations by students of their own drawings in which students were quick to correct each other if the parts were not somewhat equal. Writing the numerical unit fraction symbols helped everyone see and remember that the parts were equal even if the drawings were not totally accurate.

\subsubsection{Drawing, Numeral, and Verbal Supports to See that Adding, Subtracting, and Decomposing Unit Fractions Only Adds, Subtracts, or Decomposes the Numerators of the Fractions}

One of the most common student errors in fractions is to add/subtract the numerators and add/subtract the denominators. This error is stimulated by the vertical format of fraction symbols along with insufficient meaning-making of what those symbols mean. Writing fractions as the sum of unit fractions and seeing these unit fractions as in Figure 1 is a first step toward overcoming this typical addition/subtraction error. Students become used to writing the same unit fraction and just counting those unit fractions or adding the tops of the unit fractions to name the fraction composed from those unit fractions. Showing fraction addition and subtraction using length drawings labeled with unit fractions as in Figure 2 also helps students to build correct meanings that can resist the common error. Students can easily make and explain such drawings. They also can write the addition or subtraction (3 +2 or $6-4$ ) above a single denominator as in Figure 2 to emphasize the numerators being added or subtracted. 


\section{Add Fractions}

The circled parts of this fraction bar show an addition problem.

\begin{tabular}{|l|l|l|l|l|l|l|}
\hline$\frac{1}{7}$ & $\frac{1}{7}$ & $\frac{1}{7}$ & $\frac{1}{7}$ & $\frac{1}{7}$ & $\frac{1}{7}$ & $\frac{1}{7}$ \\
\hline
\end{tabular}

Complete this addition equation to match the problem above.

$$
\frac{3}{7}+\frac{2}{7}=\frac{3+2}{7}=\frac{5}{7}
$$

Write the numerators to complete each addition equation.

(2) $\frac{3}{9}+\frac{4}{9}=\frac{3+4}{9}=\frac{7}{9} \quad$ (3) $\frac{1}{5}+\frac{3}{5}=\frac{1+3}{5}=\frac{4}{5} \quad$ (4) $\frac{2}{8}+\frac{5}{8}=\frac{2+5}{8}=\frac{7}{8}$

5 What happens to the numerators in each equation?

The numerators are added together.

6 What happens to the denominators in each equation? The denominators stay the same.

\section{Subtract Fractions}

The circled and crossed-out parts of this fraction bar show a subtraction problem.

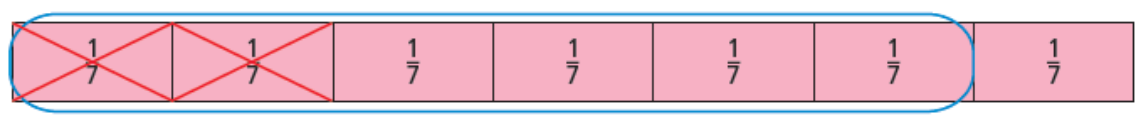

Write the numerators to complete the subtraction equation.

$$
\frac{6}{7}-\frac{2}{7}=\frac{6-2}{7}=\frac{4}{7}
$$

Figure 2. See addition and subtraction of fractions visually and with unit fractions.

Writing fractions in English words such as 3 sevenths + 2 sevenths seemed to be particularly helpful to some students because the distracting number in the denominator disappears and the words say the meaning of the fractions. Students proposed other ways to make clear that the denominator did not change, for example, circling the numerators because they tell how many and then you can just add or subtract those.

Students also decomposed 1 whole into all possible pairs of addends as shown in Figure 3. Some students had worked with such decompositions for whole numbers in kindergarten and grade 1, calling the addends partners. Some students observed that the tops of the additions showed all of the partners that made 5. Drawing and writing such decompositions was another experience in which the unit fraction (the denominator) stayed the same but the numerators added. 


\section{Fifths that Add to One}

Every afternoon, student volunteers help the school librarian put returned books back on the shelves. The librarian puts the books in equal piles on a cart.

One day, Jean and Maria found 5 equal piles on the return cart. They knew there were different ways they could share the job of reshelving the books. They drew fraction bars to help them find all the possibilities.

On each fifths bar, circle two groups of fifths to show one way Jean and Maria could share the work. (Each bar should show a different possibility.) Then complete the equation next to each bar to show their shares.

Possible answers are shown.
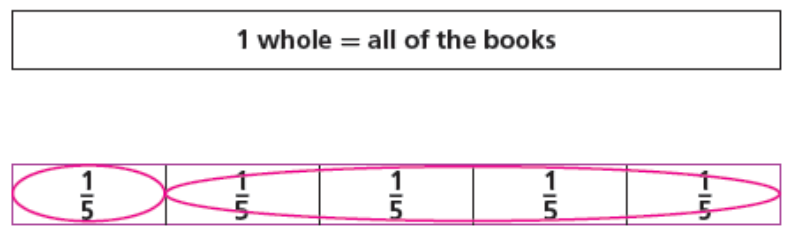

\begin{tabular}{|l|l|l|l|l|}
\hline$\frac{1}{5}$ & $\frac{1}{5}$ & $\frac{1}{5}$ & $\frac{1}{5}$ & $\frac{1}{5}$ \\
\hline
\end{tabular}
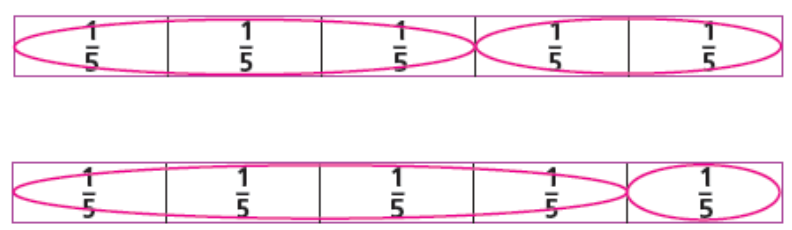

$$
\begin{gathered}
1 \text { whole Jean's } \begin{array}{c}
\text { Maria's } \\
\text { share } \\
\text { share }
\end{array} \\
\frac{5}{5}=\frac{1}{5}+\frac{4}{5} \\
\frac{5}{5}=\frac{2}{5}+\frac{3}{5} \\
\frac{5}{5}=\frac{3}{5}+\frac{2}{5} \\
\frac{5}{5}=\frac{4}{5}+\frac{1}{5}
\end{gathered}
$$

Figure 3. See the unit fraction (the denominator) stay the same but numerators add when decomposing one whole fraction as $\mathrm{n} / \mathrm{n}$

\subsubsection{Fraction Notation Shows the Number but Not the} Size of Unit Fractions in One Whole and These Are Related Inversely: A Larger Denominator Makes a Smaller Unit Fraction

The common student error when comparing different unit fractions like $1 / 3$ and $1 / 5$ (or $2 / 3$ and $2 / 5$ ) is to use whole number knowledge of which number is larger $(5>3)$ and just use that to say that $1 / 5>1 / 3$. Seeing, shading, and discussing drawings of unit fractions of the same whole such as those in Figure 4 support students in identifying the inverse relationship between the number of unit fractions shown in the denominator and the size of the unit fraction. Students readily see and describe this relationship. But fraction notation such as $1 / 3$ only shows the number of unit fractions; it does not show the size. Students must generate mental or drawn visual images for $1 / 3$ and $1 / 5$ to see that each third is larger than each fifth and so $1 / 3>1 / 5$. We found that students would compare correctly when drawings were available or they made drawings, but that whole number errors still appeared when they only worked with symbols. Quick practices every day for comparing written symbolic unit fractions in a real-world situation helped increase accuracy (remembering and using the inverse relationship). A student leader would write two unit fractions (e.g., $1 / 4$ and 1/6) and ask, "Do you want one fourth or one sixth of a sandwich/pizza/cookie?" Students also suggested ideas for how they remembered the inverse relationship: "I draw or imagine the line segment between 1 and 4 in the $1 / 4$ symbol as having 4 parts so 6 parts would be smaller." 


\section{A unit fraction has a numerator of 1 . Shade the rest of the fraction bars at the right below to represent unit fractions. What patterns do you see?}

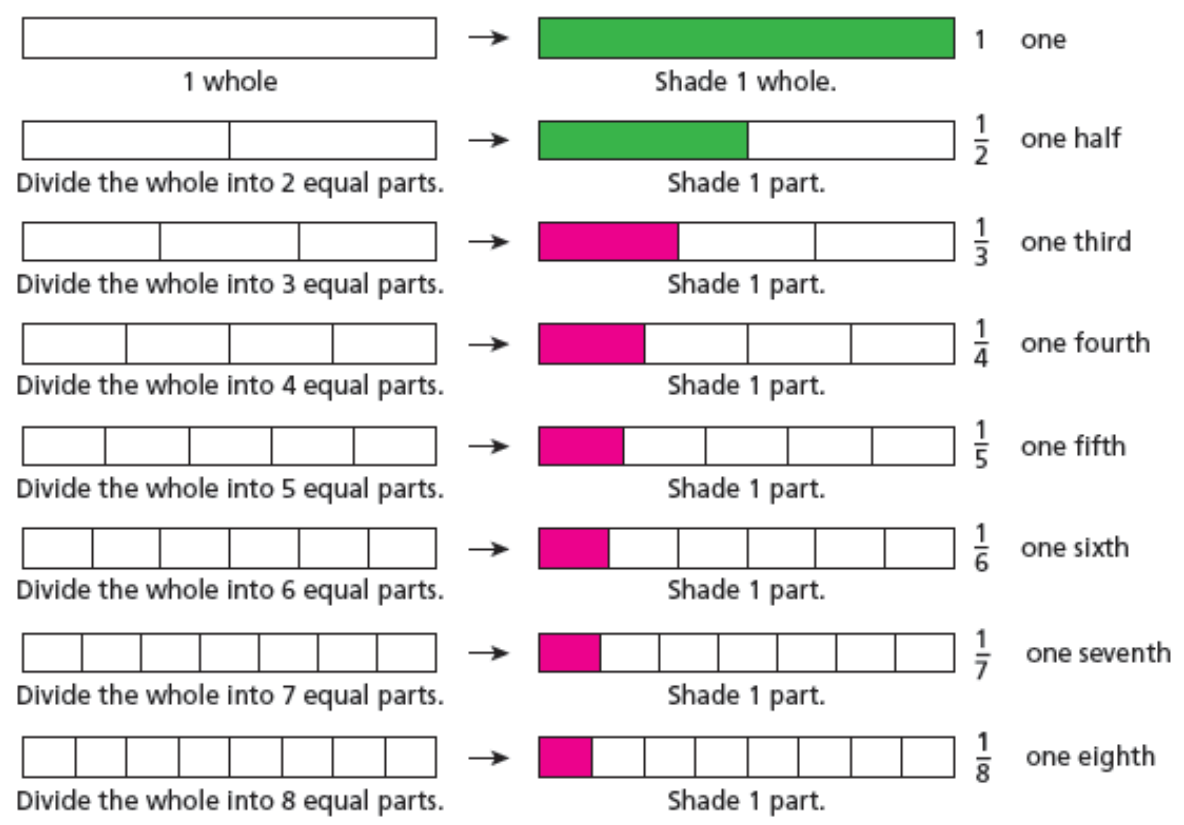

Figure 4. Fraction notation shows the number but not the size of unit fractions in one whole and these are related inversely: a larger denominator makes a smaller unit fraction

Students must differentiate two other cases of simple fraction comparisons: non-unit fractions with the same numerator but different denominator such as $2 / 5$ and $2 / 6$ and non-unit fractions with the same denominator but different numerators such as $2 / 5$ and $3 / 5$. The former case is just an extension of the work with unit fractions, and so can build on that inverse relationship. The latter case is actually easier because it only uses the meaning of a fraction as composed of unit fractions, so more unit fractions will make a larger fraction. Here student use of whole number knowledge is valid and can be explained with drawings. Mixing these two kinds of cases without drawings can become challenging, and some students always made a drawing for at least one fraction or wrote the fractions in words, such as 3 sixths versus 3 fifths.
3.2.4. For Equivalent Fractions the Fraction Notation Shows the Number of Parts Getting Greater by Multiplying but Does Not Show the Size of the Unit Fractions Getting Smaller; Visual Models Are Needed to Show the Units Getting Smaller

Finding equivalent fractions has the same difficulty as comparing fractions: The fraction notation shows the number of parts and the denominator getting greater by multiplying but does not show that the unit fractions become smaller. This inverse trade-off between the number of parts and the size of the parts is why the fractions are equivalent. These issues are illustrated in Figure 5, where looking at the multiplication at the top makes it look as if the fraction $5 / 6$ is getting larger as it changes to $10 / 12$. It takes drawings to see that the fraction $5 / 6$ stays the same total size as each 1/6 unit fraction becomes two unit fractions $1 / 12$. Figure 5 shows how a fraction bar model, a number line model, and an area model show this equivalent fraction relationship. 


\section{Equivalent Fractions}

\section{Equivalent fractions are made by:}

\section{a. more but smaller parts}

$$
\frac{5}{6}=\frac{5 \cdot 2}{6 \cdot 2}=\frac{10}{12}
$$

\section{Fraction Bar Model}

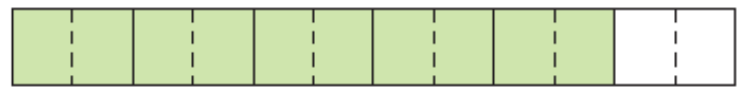

Number Line Model

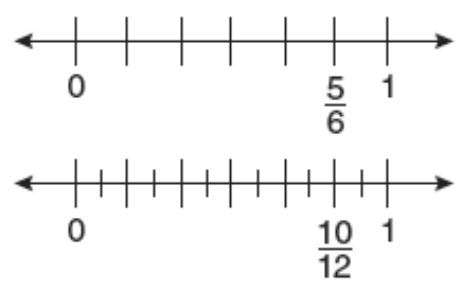

Area Model

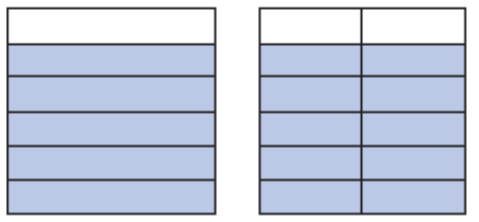

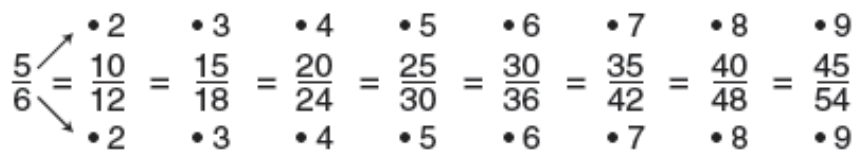

b. fewer but larger parts

$$
\frac{10}{12}=\frac{10 \div 2}{12 \div 2}=\frac{5}{6}
$$

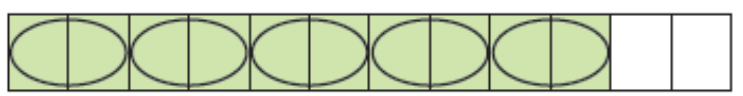

Figure 5. For equivalent fractions the fraction notation shows the number of parts getting greater by multiplying but does not show the size of the unit fractions getting smaller; visual models are needed to show the units getting smaller

The bottom of Figure 5 shows how simplifying to find an equivalent fraction also only shows the fraction numbers are getting smaller by division and does not show the unit fractions are getting larger by composing unit fractions. Many programs and teachers insist that students always simplify a fraction answer. But such simplifying gets in the way of reflecting on and seeing general patterns for computation and complicates fraction computation by adding an extra step that has little to do with that computation. We did not require always simplifying answers.

Below these models is a list of equivalent fractions made by fracturing (equal-dividing) $5 / 6$ into $2,3,4,5, \ldots$ unit fractions by multiplying the 5 and the 6 by the number above and below the new equivalent fraction. These top and bottom rows are rows from the multiplication table, with each new fraction coming from a different column (the $2 \mathrm{~s}, 3 \mathrm{~s}, 4 \mathrm{~s}$, etc. columns). Seeing such rows helped students see that making equivalent fractions was a general pattern that could go on and on multiplied by larger numbers. Students cut out rows of the multiplication table and made rows of equivalent fractions after they had made drawings to make particular equivalent fractions (see in [7] a video of this process for $2 / 7=6 / 21$ and of drawings to explain equivalence in a grade 3 classroom). Students said things like, "It makes equivalent fractions more friendly because it is just working with the multiplication table." and "To find how many 21 sts I can make from $2 / 7$, I know both top and bottom numbers will be in the 3 column because 7 times 3 is 21 ."

When adding, subtracting, or comparing fractions with different denominators, students first have to remember that they have to work with the same unit fractions. After the above approaches, this is fairly easy for most students to remember. But then they have to decide which equivalent fractions to make from the two given fractions. There are three cases: 
1) one denominator divides the other denominator such as $2 / 3$ and $5 / 9$;

2) the denominators are relatively prime such as $2 / 3$ and $4 / 5$;

3) the denominators are each factors of a larger denominator such as $3 / 8$ and $5 / 6$.

In the first case, the larger denominator is an easy common denominator. In the second case, the common denominator is the product of the two denominators. For the third case, it is often the easiest for many students to use the same approach-using the product of the two denominators. This greatly simplifies the decision about which common denominator to use, and the numbers usually do not get so large to be a disadvantage. We suggested that students in the third case see if they can think of a smaller number that is a product of both denominators such as 24 but use the product 48 if they cannot easily think of a number. Deciding a common denominator was the difficult part for students, and we see no need to insist that they use the smallest common denominator.

A small technical point about equivalent fractions is that when you multiply the top and bottom of a fraction by the same number $n$, you are multiplying by $n / n$, which is 1 so you are not changing the amount of the fraction. But if multiplying fractions is done after finding equivalent fractions, as in most programs, you can't use this argument and must rely on generalizing from examples with drawings.

\subsubsection{Students Need Support to See the Lengths in} Number Lines and that Number Line Numbers Tell the Number of Unit Fractions from Zero

Number lines for whole numbers are difficult for students and fractions are even more difficult. Students are used to seeing and counting things, so they look at the marks instead of the lengths on number lines and so are off by one. U.S. National Research Council reports [e.g., 8] recommend that students first work with number lines in grade 2 (age 7 to 8 ) when they begin measuring length with rulers. Rulers can use simple whole numbers as in Figure 6. I found that having students draw a length of $1 \mathrm{~cm}$, then just below a length of $2 \mathrm{~cm}$, etc. as shown in Figure 6 can help students understand what a ruler is and see that the numbers tell how many lengths so far. Students would say things like, "Look, if we push down the little lines we drew, we get a ruler!!!" and "The numbers say how many centimeters we have drawn." Having students draw and mark unit lengths on rectangles or line segments helps them focus on the lengths, as does moving a finger along each length as it is counted.

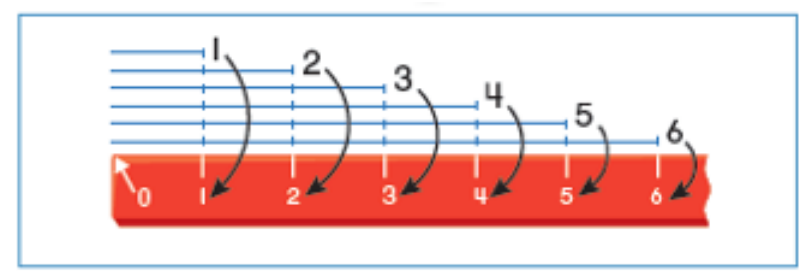

Figure 6. Drawing length units to see how a ruler labels the number of lengths so far

Students have similar problems with fraction number lines. They draw too many or too few segments and count the marks or the fraction symbols instead of the lengths. Figure 7 shows two ways to help students to see the lengths in a number line. A fraction bar drawing can be put above the number line so that the lengths in the bars help make the lengths in the number line more salient. Encircling the lengths on the fraction number line also draws student attention to the lengths so that they see that $7 / 4$ is 7 of the unit fraction $1 / 4$ lengths from 0 rather than $8 / 4$ which some students get by counting the little vertical marks or the numbers starting with 0 . 


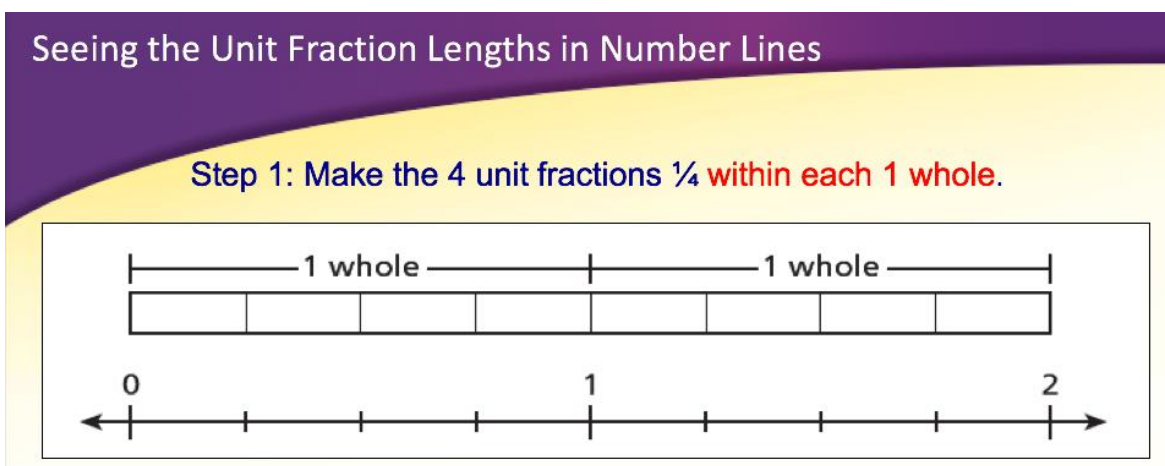

Step 2: Shade or encircle 7 unit fractions and label the number line.

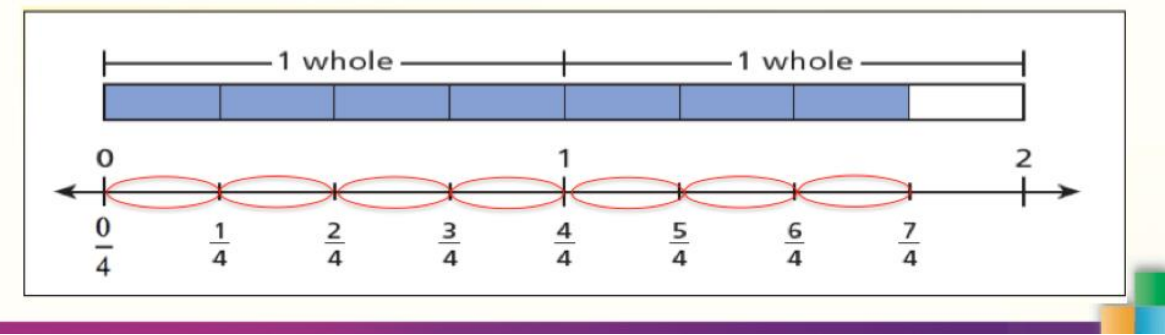

Figure 7. Support to see the unit fraction lengths in number lines

\section{Understand Tenths and Hundredths}

Answer the questions about the bars and number lines below.
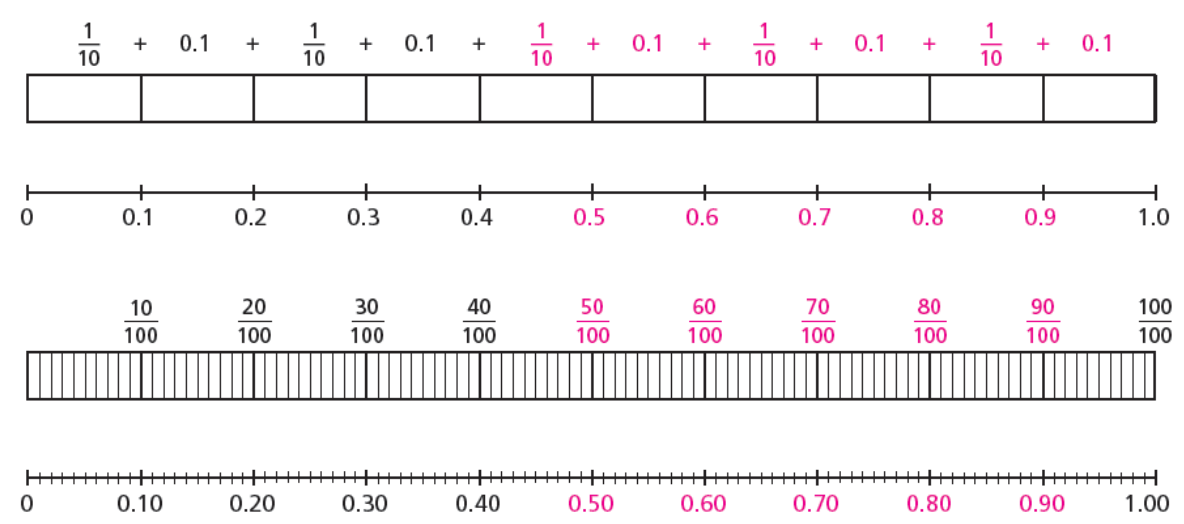

Figure 8. Seeing that number line numbers tell the number of unit fraction lengths from zero

In Figure 8, students can see fraction and decimal bar models and number lines that help students see the lengths in all models by placing them above each other. Students can also discuss the different ways that the models are labeled symbolically. In the top bar model the symbolic unit fractions and unit decimals are centered above each length so that each unit is labeled; this draws attention to the lengths and students can see clearly what the lengths represent. The bottom three models use number line labels that tell the number of unit fraction or unit decimal lengths from zero. These labelled points are the end-points of lengths from zero. Students can discuss these different ways and what each shows, and encircle or run a finger along lengths to make or check this summative number line labelling.

\subsubsection{Bar, Number Line, and Area Models Can Show Why a Fraction Times a Fraction is the Product of the Numerators over the Product of the Denominators (the Unit Fractions)}

In the first study [6] students initially drew their own length models for a simple problem of a unit fraction of a whole number in which the unit fraction divided the whole number: $1 / 3 \times 6$. Most of the students took the whole 
number as a single magnitude length 6 and divided it into 3 equal parts to find $1 / 3$ of 6 was 2 . Some of the students took $1 / 3$ of a length of 1 which was $1 / 3$ and did that 6 times to take $1 / 3$ of 6 and so had 6 thirds which was 2 . Then we gave a problem in which the unit fraction did not divide the whole number evenly. Students using the first method could not solve such problems, while the second method did generalize to any problem of a fraction times a whole number and then to a fraction times a fraction. So it is important to give students problems that will stimulate general solutions and not spend a lot of time on simpler problems that will elicit special solutions that will not generalize.

Over the three studies, students drew bar models, number lines, or area models (and sometimes all three models) to show and explain a fraction times a fraction using the general "of each piece" approach. Examples of each with student explanations are shown in Figure 9. Students show on the drawing the fraction being multiplied (here 4/5) and then divide each of those unit fractions (fifths) into thirds to get ready to take $2 / 3$ of each fifth. This makes new smaller unit fractions of fifteenths. So students can see that this step will always involve multiplying the denominators. Students then take $2 / 3$ of each of the 4 fifths $(4 / 5)$, which results in 4 groups of $2 / 15^{\text {ths }}$ which is multiplying the numerators 2 and 4 to make 8 . A video of a fifth grade classroom in which two different students explain this process with a number line model is in [9]. A student then explains how these models show that you can multiply numerators and multiply denominators to find the product of two fractions. The teacher then leads a discussion of how you can use the Commutative Property for whole numbers to see that this property holds for fractions.

\section{Miguel explains how to use fraction bars to find $\frac{2}{3} \cdot \frac{4}{5}$ :}

First, I circle 4 fifths on the fifths fraction bar.
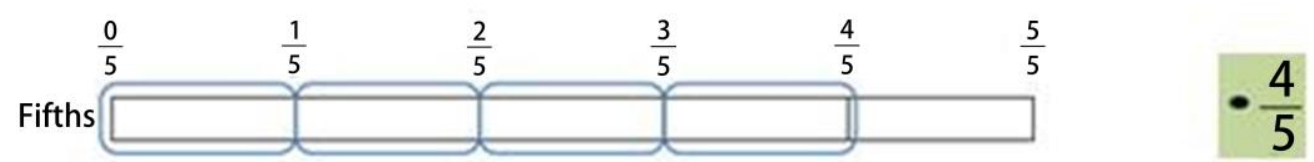

To find $\frac{2}{3}$ of $\frac{4}{5}$, I can circle $\frac{2}{3}$ of each fifth. But, first I have to split each fifth into three parts. After I do this, the bar is divided into fifteenths.
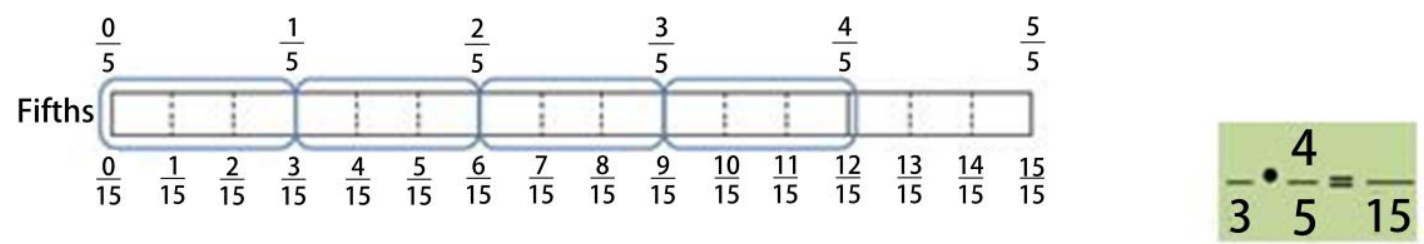

\section{Now, it is easy to circle 2 thirds of each of the 4 fifths.}

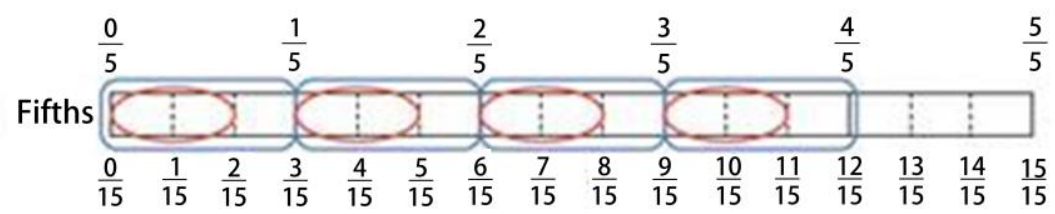

\section{Each group I circled has 2 fifteenths, so I circled 4 groups of}

2 fifteenths. That's 8 fifteenths in all. So, $\frac{2}{3} \cdot \frac{4}{5}=\frac{8}{15}$.

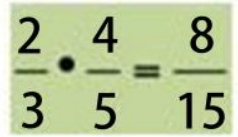




$$
\frac{2}{3} \cdot \frac{4}{5}=\frac{2 \cdot 4}{3 \cdot 5}=\frac{8}{15}
$$

Ginny's Model "I use a number line. First I model $\frac{4}{5}$ (red loop), and then I separate each fifth into 3 equal parts, or thirds (green tick marks). This makes 15 equal parts altogether. Then I take $\frac{2}{3}$ of each fifth (blue loops), which is $\frac{8}{15}$."

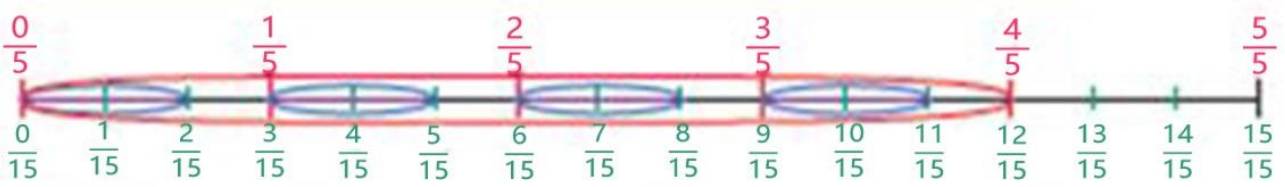

\begin{abstract}
Taylor's Model "I use an area model. First, I model $\frac{4}{5}$ by dividing the area of a square into fifths and shading 4 of them red. To find $\frac{2}{3}$ of those 4 fifths, I divide the area into thirds and shade 2 of them blue. The whole area is divided into fifteenths, and there are 8 fifteenths in which the shading overlaps."
\end{abstract}

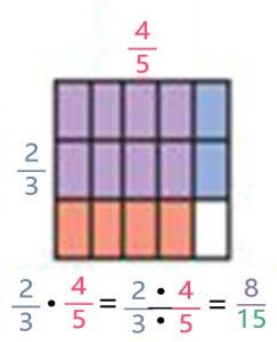

Figure 9. Bar, number line, and area models can show why a fraction times a fraction is the product of the numerators over the product of the denominators (the unit fractions)

I prefer the length models over the area model because students have to understand and actively use the $2 / 3$ of each $4 / 5$ meaning. In the area model this "of each" meaning is passively done by the area model as you draw the horizontal segment across the fifths. We did find that the area model was easier for problems in which the numerator was greater than the denominator because it showed the parts greater than one more easily.

A recurring issue for multiplication of fractions is why does "of" mean "times"? Students had no trouble understanding "one-third of 6" as dividing 6 into 3 equal parts, but this experience then made them think that they were dividing, not multiplying. In fact, multiplying by a unit fraction is divided by that whole number, but the operation within fractions is multiplication. Either multiplication grouping or comparing language can provide a basis for understanding why "of" means times for fractions as well as for whole numbers. Across different countries, people interpret $4 \times 2$ in two ways: as " 4 sets of 2 " or as "a set of 4 taken 2 times." Using both of these meanings relates the words "of" and "times" within the English language. So $4 / 5 \times 2 / 3$ can mean " $4 / 5$ of $2 / 3$ " or " $4 / 5$ taken 2/3 times." Within multiplicative comparing situations, English shifts from the "times as many" to "fractional parts of" language, again providing a relationship between these two. For example, we say, "Joe has 3 times as much as Mary has" but "Mary has $1 / 3$ of Joe's amount."

\subsubsection{Division as Finding the Unknown Factor: You can Divide Fractions by Dividing the Numerators and Dividing the Denominators}

Division of fractions is often identified as one of the most difficult and mysterious aspects of computation of any numbers, because the computation method is often taught to multiply the product by the reciprocal of the known factor: $8 / 15 \div 2 / 3=8 / 15 \times 3 / 2$ and this is difficult to relate to division or to what students know about multiplication of fractions. But in fact, if you take any two fractions and multiply them, you can solve the two related division problems in the sensible way: you divide the numerators and divide the denominators:

If $3 / 5 \times 10 / 11=30 / 55$, then $30 / 55 \div 10 / 11=3 / 5$ and $30 / 55$ $\div 3 / 5=10 / 11$

This is true for any two fractions: You can always find the related divisions in this sensible way. So division of fractions is just what you would want and expect!

This numerical process is so straightforward that you don't even have to make a drawing. But a drawing is given in Figure 10 for finding the unknown factor for a division problem $8 / 15 \div 2 / 3$ : How many $2 / 3$ cup servings are in $8 / 15$ of a pot of hot chocolate? 
2 The mugs at a restaurant hold $\frac{2}{3}$ cup of hot chocolate.

The restaurant has $\frac{8}{15}$ cup hot chocolate left in its pot.

How many servings of $\frac{2}{3}$ cup are in the pot?

$\frac{4}{5}$ serving

Step 1 Write an equation.

$\frac{?}{?} \cdot \frac{2}{3}=\frac{8}{15}$

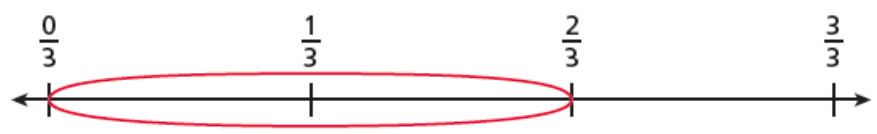

Step 2 Look at the denominators.

Divide each $\frac{1}{3}$ into 5 equal parts to make fifteenths.

$\frac{?}{5} \cdot \frac{2}{3}=\frac{8}{15}$

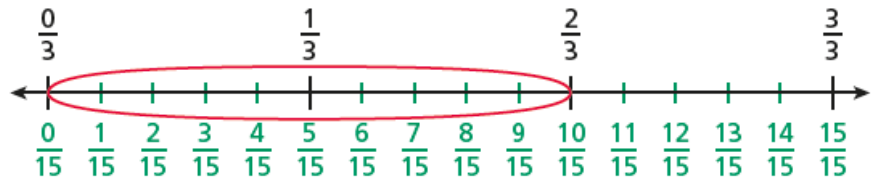

Step 3 Look at the numerators.

Take 4 fifteenths from each of the 2 thirds to make $\frac{8}{15}$.

$\frac{4}{5} \cdot \frac{2}{3}=\frac{8}{15}$

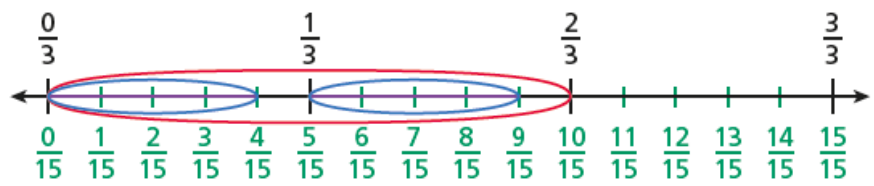

Figure 10. Division as finding the unknown factor: You can divide fractions by dividing the numerators and dividing the denominators

\section{Unsimplify to Divide}

$\frac{2}{3} \div \frac{5}{7}=?$

We cannot divide the numerator of $\frac{2}{3}$ by 5 or the denominator by 7 .

To be able to divide, we need to unsimplify $\frac{2}{3}$. To unsimplify we rewrite it as an equivalent fraction so the numerators and denominators

divide evenly.

Step $1 \frac{2}{3} \div \frac{5}{7}=\underbrace{\frac{2}{3} \cdot 1 \cdot 1}_{\left.\frac{2}{\frac{2}{3} \cdot \frac{5}{5} \cdot \frac{7}{7}}\right)} \div \frac{5}{7}$

Step $2=\frac{2 \cdot \overbrace{5 \cdot 5=1}^{7}+\frac{5}{7}}{3 \cdot 5 \cdot 7}=1$

(1) How is the number you divide $\frac{2}{3}$ by in the original division problem related to the number you multiply $\frac{2}{3}$ by in the final multiplication problem?

Step $3=\frac{2 \cdot 7}{3 \cdot 5}$

You multiply by $\frac{7}{5}$, the reciprocal

of original divisor, $\frac{5}{7}$.

Step $4=\frac{2}{3} \cdot \frac{7}{5}$

Figure 11. When the numerator and denominator of the known factor do not divide the numerator and denominator of the product, unsimplify the product so that you can divide and notice that this is the same as multiplying the product by the reciprocal of the known factor

3.2.8. When the Numerator and Denominator of the Known Factor Do Not Divide the Numerator and Denominator of the Product Because It Has Been Simplified, Unsimplify the Product So That You Can Divide and Notice that This Is the Same as Multiplying the Product by the Reciprocal of the

\section{Known Factor}

So what is the difficulty for fraction division? The difficulty is that fractions are often simplified and then the numerator cannot divide the numerator or the denominator cannot divide the denominator or both. For example, $70 / 105 \div 5 / 7=14 / 15$ by dividing numerators and 
denominators. But if $70 / 105$ is simplified by dividing by $35 / 35$, the division problem becomes $2 / 3 \div 5 / 7$ and 2 is not divisible by 5 and 3 is not divisible by 7 . What to do? Well, we say in our classrooms, "If some mean person simplified the product so you can't divide, why don't you unsimplify that product so that you can divide." This process is shown in Figure 11.

A typical student explanation for Figure 11 is,

"I know I want to divide the top by 5 because $5 / 7$ has 5 in the top. But I can't just multiply $2 / 3$ by 5 because that will change its value. So I have to multiply by $5 / 5$ which is 1 . And I want to divide the bottom by 7 because $5 / 7$ has a 7 here in the bottom, so I also have to multiply $2 / 3$ by $7 / 7$. So here in these parentheses is my $2 / 3$ unsimplified. But I don't have to multiply it all out because I am going to divide the top by 5 and the bottom by 7 first. Look what happens when I do that. See here in Step 2 the 5 in the unsimplified $2 / 3$ becomes 1 because $5 \div 5=1$. And, when I divide the bottom by 7 , the 7 in the unsimplified $2 / 3$ becomes 1 . So I have 2 times 7 all over 3 times 5 . But that is just $2 / 3$ times $7 / 5$.

$$
\frac{2 \cdot 7}{3 \cdot 5}=\frac{2}{3} \cdot \frac{7}{5}
$$

So here is the pattern: $\frac{2}{3} \div \frac{5}{7}=\frac{2}{3} \cdot \frac{7}{5}$

You get the same thing if you multiply the original product $2 / 3$ by the known factor $5 / 7$ flipped over to be $7 / 5$. This is because you multiplied $2 / 3$ by $5 / 5$ and $7 / 7$ to unsimplify it and then divided by 5 on the top and 7 on the bottom, so you have flipped the known factor over. We call this flipped factor the reciprocal."

Some students could give such a full explanation, most could give parts of it independently, and all could give a full explanation if helped by questions from other students or the teacher.

Students then did other examples to check to see if this worked for the cases where they only needed to unsimplifty by multiplying the numerator or by being multiplied by the denominator. They then discussed what method they would use for different kinds of division of fraction problems. All said they would divide tops and bottoms if they could. Most said they would multiply the product by the reciprocal if they could not divide both tops and bottoms, but a few students would unsimplify the product if they only had to unsimplify the numerator or the denominator. We discussed how to remember which number to flip, the product or the known factor, and students said they would say "flip the factor" to remember this. Some would even say, "I flip the factor because that's what I get when I unsimplify the product."

Throughout the work with fractions, we accept students saying top number and bottom number instead of numerator and denominator because those terms make more sense and help direct everyone's attention to the correct place in the symbol (the top and the bottom). Of course, students need to understand what numerator and denominator mean, but requiring their use at all times adds unnecessary complication to sense-making discussions, especially for students not fluent in English. We took the same approach to using the term reciprocal. Students need to know what it means and be able to find the reciprocal, but they can explain their process for problems in which the tops and bottoms do not divide the product by using the mnemonic flip the factor. To make it easier for students to communicate and to emphasize the relationship between addition and subtraction and between multiplication and division, we used the terms for parts of an addition equation (addend, addend, total) for subtraction and the parts of a multiplication equation (factor, factor, product) for division. We have done so in this paper also. We used in the classroom the word total instead of sum because English has this terrible confusion between sum (the total) and some (an addend) that are pronounced the same. Also total relates to the Spanish word total, so this word was easier for the many students from Spanish-speaking backgrounds.

3.2.9. Fraction Operations Have the Same Meanings as Operations with Whole Numbers, but the Results of Multiplying or Dividing by a Fraction less than One Are Opposite to such Results with Whole Numbers

Addition and subtraction of fractions have the same situational meanings as addition and subtraction of whole numbers: Add To/Take From, Put Together/Take Apart, and Additive Compare situations. These operations also have the same results: adding results in a total larger than either addend or subtracting results in an addend less than the total. Comparing to find which is greater/less than also has the same meanings and results for fractions and whole numbers once fractions are expressed in the same unit fraction. But fraction symbols and words that do not support fraction meanings stimulate errors by showing only the number of unit fractions in the denominator and not showing the size of those unit fractions. Drawings are needed to show that the size of the unit fraction gets smaller as the number of unit fractions gets larger (see Figure 4). Adding, subtracting, and comparing require that fractions be expressed in the same units, i.e., the same unit fractions.

Multiplying and dividing of fractions also have the same situational meanings as multiplying and dividing of whole numbers: Equal Groups, Arrays/Area, and Multiplicative Compare situations. But for fractions less than one, any of the multiplication situational meanings result in a product less than the factor being multiplied, for example, $12 / 13 \times 9 / 5$ will be less than $9 / 5$ because you will divide each of the $1 / 5$ into 13 equal parts but take only 12 of those parts. This is a result different from that for whole numbers where multiplying always results in a product larger than the factor being multiplied. Division by a fraction less than one also has a result opposite to that for whole numbers. Division by a whole number results in a number smaller than the dividend (the product) because you are finding the 
number of groups greater than one in that dividend. Division by a fraction less than one results in a factor greater than the product because the groups being embedded in the product are smaller than one so there will be more than one in each whole and therefore more groups in the product than in the product number.

Teachers need to discuss the above points with students so that these patterns are clear. Students need to work with problem sets that mix operations to continue to resist errors. For example, students may not add or subtract tops and bottoms of fractions initially but may begin to do so after they have been multiplied by multiplying tops and bottoms of fractions. Continual sense-making in the ways outlined in this paper, and in ways elicited from and suggested by students, is needed to escape the seductive errors stimulated by fraction notation in the absence of sense-making.

\section{Discussion}

We have focused here just on the parts of teaching and learning fractions that are the most problematic. A complete approach also provides experience with models other than length or area models and includes word problems that students solve and write. Differentiating the situation operation from a solution operation is particularly important for word problems. For example,
"How many helpings of 1/3 cup can Sergio serve from his recipe for 2 cups of rice pudding?" is a division situation in which the product 2 cups and one factor $1 / 3$ cup are known, but the solution involves multiplying 3 (cups in 1 whole cup) times 2 cups. More details about the methods and drawings summarized here and other related issues with fractions are in [10].

The teaching experiment research design was intended to find out whether length drawings and Math Talk that clarified the roles of unit fractions in computation could be successful with a range of students including those from non-English-speaking homes and backgrounds of poverty. Results from East Asian students were included as a base-line comparison because they generally show higher levels of fraction performance than students in the United States do and so can indicate what fifth graders can do. More focused research studies are needed to untangle aspects of language, culture, and teaching that are responsible for these differences in performance. But our results do indicate that such differences can be reduced by effective teaching approaches.

Our results support the use of the three general design principles and the nine more specific design issues about specific fraction meanings or operations listed in Table 1. The latter nine were each discussed in sections 3.2.1 through 3.2.9 as ways to support understandings about and reducing specific kinds of errors.

Table 1. Design Principles and Issues for Teaching Fraction Computation with Understanding

\begin{tabular}{|l|}
\hline \multicolumn{1}{|c|}{ General design principles about all computations } \\
\hline Unit fractions $1 / n$ compose to make any fraction and are the basis of all computation with fractions. \\
\hline Length models support all fraction computations by supporting students to explain how unit fractions work in each kind of computation. \\
\hline A Math Talk Community in which students make drawings and explain their thinking supports meaning-making and reduces errors. \\
\hline \multicolumn{1}{|c|}{ Design issues about specific fraction meanings or operations } \\
\hline $\begin{array}{l}\text { See unit fractions visually and in fraction notation } 1 / n \text { by showing division of the whole into n equal parts and then in a separate drawing } \\
\text { composing any fraction by shading or circling these unit fractions. }\end{array}$ \\
\hline $\begin{array}{l}\text { Drawing, numeral, and verbal supports to see that adding, subtracting, and decomposing unit fractions only adds, subtracts, or decomposes } \\
\text { the numerators of the fractions. }\end{array}$ \\
\hline $\begin{array}{l}\text { Fraction notation shows the number but not the size of unit fractions in one whole and these are related inversely: a larger denominator } \\
\text { makes a smaller unit fraction. }\end{array}$ \\
\hline $\begin{array}{l}\text { For equivalent fractions the fraction notation shows the number of parts getting greater by multiplying but does not show the size of the unit } \\
\text { fractions getting smaller; visual models are needed to show the units getting smaller. }\end{array}$ \\
\hline Students need support to see the lengths in number lines and that number line numbers tell the number of unit fractions from zero. \\
\hline $\begin{array}{l}\text { Bar, number line, and area models can show why a fraction times a fraction is the product of the numerators over the product of the } \\
\text { denominators (the unit fractions). }\end{array}$ \\
\hline Division as finding the unknown factor: You can divide fractions by dividing the numerators and dividing the denominators. \\
\hline $\begin{array}{l}\text { When the numerator and denominator of the known factor do not divide the numerator and denominator of the product because it has been } \\
\text { simplified, unsimplify the product so that you can divide and notice that this is the same as multiplying the product by the reciprocal of the } \\
\text { known factor }\end{array}$ \\
\hline $\begin{array}{l}\text { Fraction operations have the same meanings as operations with whole numbers, but the results of multiplying or dividing by a fraction less } \\
\text { than one are opposite to such results with whole numbers. }\end{array}$ \\
\hline
\end{tabular}


We see three phases in building understandings in such a complex domain. First, the domain is approached using intuitive easy numbers in situations where objects or drawings can help students develop meanings. Second, these meanings are connected to generalizable numerical methods through discussion and linking to drawings. Third, a longer period follows of remembering and explaining in which occasional practice with mixed numeric methods by students is accompanied by explaining why the method works. This phase is required to keep the fraction meanings connected to the general numeric meanings and to maintain the relationships among various computations with fractions and whole numbers as summarized in 3.2.9. The first phase must be done with a view to the second and third phases because we saw in [6] how the use of easy intuitive numbers that divided easily led students to methods of multiplying by a unit fraction that did not generalize to numbers that were not evenly divisible. Curriculum development must keep all of these phases in mind from the beginning if students are not to be led to develop methods that will not generalize.

The approaches summarized here are used in a kindergarten through grade 6 math program used in every state in the United States and in some Latin American countries [11]. The approaches have been successful in the wide range of backgrounds and languages students and have been brought into classrooms in the United States. The points made above about fraction words in English may not generalize to all languages, but linguistic methods can be sought for any language to emphasize the equal dividing to make unit fractions and then taking the number of such unit fraction to make a given fraction. These approaches are not the only ones that can reduce errors and increase understanding, but they are powerful and should be considered by teachers, schools, and developers of math programs or technology for schools.

\section{Conclusions}

Students and teachers can understand and become able to explain fraction arithmetic-methods of comparing, finding equivalent fractions, adding, subtracting, multiplying, and dividing. Sense-making in the ways discussed here can reduce typical errors students make and engender understanding.

\section{Acknowledgements}

Thanks to the teachers, students, and members of the Children's Math Worlds design research team. This research was funded by the National Science Foundation under grant RED-935373.

\section{REFERENCES}

[1] J. W. Stigler, S. Y. Lee, H. W. Stevenson. The Mathematical Knowledge of Japanese, Chinese, and American Elementary School Children, National Council of Teachers of Mathematics, Reston, VA.,1990.

[2] M. J. Behr, G. Harel, T. R. Post, R. Lesh. Rational number, ratio, and proportion. In Handbook of Research on Mathematics Teaching and Learning, 296-333, Macmillan, New York, 1992.

[3] R. S. Siegler, D. W., Braithwaite. Numerical development, Annual Review of Psychology, 68, 187-213, 2017.

[4] K. C. Fuson (2009). Avoiding misinterpretations of Piaget and Vygotsky: Mathematical teaching without learning, learning without teaching, or helpful learning-path teaching? Cognitive Development, 24(4), 343-361. doi:10.1016/j.co gdev.2009.09.009

[5] K. C. Fuson, A. Murata, \& D. Abrahamson. Using learning path research to balance mathematics education: Teaching/learning for understanding and fluency. In R. Cohen Kadosh \& A. Dowker (Eds.), Oxford handbook of numerical cognition (pp. 1036-1054). Oxford, UK: Oxford University Press, 2015. doi: 10.1093/oxfordhb/978019964 2342.013.003

[6] K. C. Fuson, M. Kalchman. A Length Model of Fractions Puts Multiplication of Fractions in the Learning Zone of Fifth Graders. In D. L. Haury Ed, Proceeding of the Twenty-Fourth Annual Meeting of the North American Chapter of the International Group of the Psychology of Mathematics Education, ERIC Clearinghouse for Science, Mathematics, and Environmental Education, Columbus, OH., 1641-1649, 2002.

[7] In http://www.karenfusonmath.com click on MENU then Classroom Videos then H G3 \& G5 Fractions and Ratios then Part 1.

[8] C. T. Cross, T. A. Woods, \& H. Schweingruber, H. (Eds.). Mathematics learning in early childhood: Paths toward excellence and equity. Washington, DC: National Academies Press, 2009. doi:10.17226/12519

[9] In http://www.karenfusonmath.com click on MENU then Classroom Videos then $\mathrm{C}$ then Part 5.

[10] In http://www.karenfusonmath.com click on MENU then on Teaching Progressions then on Math Expressions and NF Number Fractions Parts 1 through 4.

[11] K. C. Fuson. Math Expressions, Kindergarten through Grade 6. Houghton Mifflin Harcourt, Boston, MA, 2006, 2009, 2013, 2018. 